\title{
Improving Interactions between a Power-Assist Robot System and Its Human User in Horizontal Transfer of Objects Using a Novel Adaptive Control Method
}

\author{
S. M. Mizanoor Rahman ${ }^{1}$ and Ryojun Ikeura ${ }^{2}$ \\ ${ }^{1}$ Institute for Media Innovation, Nanyang Technological University, 50 Nanyang Drive, Singapore 637553 \\ ${ }^{2}$ Division of Mechanical Engineering, Graduate School of Engineering, Mie University, Tsu, Mie 514-8507, Japan \\ Correspondence should be addressed to S. M. Mizanoor Rahman, mizansm@hotmail.com
}

Received 31 March 2012; Revised 15 October 2012; Accepted 9 November 2012

Academic Editor: Cathy Bodine

Copyright ( $) 2012$ S. M. M. Rahman and R. Ikeura. This is an open access article distributed under the Creative Commons Attribution License, which permits unrestricted use, distribution, and reproduction in any medium, provided the original work is properly cited.

\begin{abstract}
Power assist systems are usually used for rehabilitation, healthcare, and so forth.This paper puts emphasis on the use of power assist systems for object transfer and thus brings a novelty in the power-assist applications. However, the interactions between the systems and the human users are usually not satisfactory because human features are not included in the control design. In this paper, we present the development of a 1-DOF power assist system for horizontal transfer of objects. We included human features such as weight perception in the system dynamics and control. We then simulated the system using MATLAB/Simulink for transferring objects with it and (i) determined the optimum maneuverability conditions for object transfer, (ii) determined psychophysical relationships between actual and perceived weights, and (iii) analyzed load forces and motion features. We then used the findings to design a novel adaptive control scheme to improve the interactions between the user and the system. We implemented the novel control (simulated the system again using the novel control), the subjects evaluated the system, and the results showed that the novel control reduced the excessive load forces and accelerations and thus improved the human-system interactions in terms of maneuverability, safety, and so forth. Finally, we proposed to use the findings to develop power assist systems for manipulating heavy objects in industries that may improve interactions between the systems and the users.
\end{abstract}

\section{Introduction}

Power assist system is a human-robot system that augments human's abilities and skills in performing tasks [1]. Breakthrough in power assist systems was conceived in early 1960s with "Man-amplifier" and "Hardiman", but the research on this promising field is not so satisfactory yet [1]. Currently, power assist systems are developed mainly for the sick, disabled and old people as rehabilitation and healthcare assistance $[2,3]$. A few power assist devices have also been developed for other applications for example, lifting baby carriage [4], supporting agricultural works [5], hydraulic power-assist for automobiles [6], skill-assist in manufacturing [7], power-assisted slide doors for automobiles [8], power-assisted control for bicycle [9], power assistance for sports and horse training $[10,11]$, and so forth.
We think that handling heavy objects, which is common and necessary in industries, may be another potential field of application of the power assist systems [12, 13]. It is very necessary to move heavy objects in industries such as manufacturing and assembly, logistics and transport, construction, mining, disaster and rescue works, forestry, agriculture, and so forth. Manual handling of heavy objects is very tedious and it causes work-related disabilities and disorders such as back pain to the humans [14].

On the other hand, autonomous devices may not provide desired flexibility in object handling and positioning in many cases [15]. Hence, it is thought that the uses of suitable power assist systems may be appropriate for handling heavy objects in the aforementioned industries. However, such power assist systems are not found in the industries as their design has not got much attention yet [13]. 
Again, a power assist system reduces the perceived heaviness of an object handled with it [1], and hence, the load force (manipulative force tangential to grip surfaces) required to handle an object with power-assist should be lower than that required to handle the object manually. But, the limitations with the conventional power assist devices are that the human operator cannot perceive the heaviness of the object correctly before handling it with the assist system and eventually applies excessive load force, which results in faulty interactions between the system and the user such as sudden increase in acceleration, fearfulness of the user, lack of maneuverability and stability, fatal accident, and so forth [13]. However, the conventional assist devices for object manipulation do not consider this issue [16-19].

There are also other limitations in the conventional power assist devices for object manipulation as follows: human features are not included in control, the system is itself heavy, the amount of power assistance is unclear, the system is not evaluated properly for safety, maneuverability, efficiency, and so forth [16]. Again, the system may have the disadvantages of pneumatics, hydraulics, and so forth [16]. Operator's intention is not reflected in the control, and the system generates vibrations [17]. Human force is not measured directly and separately, the system restricts movement due to constraints, there are difficulties in path planning, the object handling speed is slow, and so forth [18]. Sometimes, the system generates excessive power [19]. Moreover, there are some common problems/issues with power assist devices such as actuator saturation, noises and disturbances, adjustment with human users, selection of appropriate control methods, accuracy, capacity and sensitivity of force sensors, number of force sensors, configuration of force sensors and of the entire system, number of degrees of freedom, stability, and so forth that should be addressed. However, the conventional power assist devices do not adopt any holistic approach to address these problems/issues to make the systems human-friendly.

In the industries, the workers need to manipulate objects in different directions such as vertical lifting (lifting objects from lower position to higher position) [13], vertical lowering (lowering objects from higher position to lower position), horizontal transfer [17], and so forth in order to fulfill the task requirements [13]. We assume that the maneuverability, heaviness perception, forces and motion features, task requirements, and so forth for manipulating objects with power-assist among these directions may be different from each other and these differences may affect the control and the system performances. Hence, it seems to be necessary to study the object manipulation with power-assist in all these directions, compare them to each other, and to reflect the differences in the control design. However, such study has also not been carried out yet.

We studied the lifting of objects in the vertical direction in our previous works [13], but transferring objects in the horizontal direction is still unaddressed though the horizontal transfer of objects is very necessary in many practical cases in the industries. A few researches studied the power assist devices for transferring objects in the horizontal direction $[17,20]$. But, these devices are not designed targeting the industrial applications and these devices have limitations in their performances as mentioned above because human features are not included in their controls, that is, the biomimetic approach of the control design is ignored.

Our pioneering research addresses the aforementioned issues holistically and aims to develop a model of power assist device to handle heavy objects that does not have the above limitations $[12,13]$. This paper, as a part of the entire research, presents the design and evaluation of a novel adaptive control scheme for transferring objects with powerassist horizontally in cooperation with the humans based on human features that improves the human-system interactions. We developed a 1-DOF power assist system for transferring objects horizontally by the human subjects. We included weight perception in the system dynamics and control. We simulated the system and determined the optimum maneuverability conditions for transferring objects. We determined the psychophysical relationships between actual and perceived weights and analyzed the load forces and motion features for transferring objects with the system. We then used the human features to design, implement and evaluate a novel adaptive control scheme to reduce excessive load forces and accelerations, and thus to improve the performances of the human-robot system.

The results showed that the novel control improved the human-system interactions in terms of safety, maneuverability, naturalness, and so forth. We compared the findings for the horizontal transfer of objects with power-assist to that for the vertical lifting of objects [13]. Finally, we proposed to use the findings to develop human-friendly power assist devices for handling heavy objects in industries that may enhance interactions with human users in terms of maneuverability, safety, naturalness, and so forth.

\section{The Experimental Human-Robot System}

2.1. System Configuration. We developed a 1-DOF (horizontal translational motion) power assist robot system using a ball screw assembly actuated by an AC servomotor (Type: SGML-01BF12, made by Yaskawa, Japan). We coaxially fixed the ball screw and the servomotor on a metal plate and horizontally placed the plate on a table. We made three rectangular boxes by bending aluminum sheets (thickness: $0.5 \mathrm{~mm}$ ). The boxes were horizontally transferred with the system by the human subjects and were called the powerassisted objects (PAOs). A PAO (box) could be tied to the ball nut through a force sensor (foil strain gauge type, NEC Ltd.) and be transferred by a subject. The dimensions (length $\times$ width $\times$ height) of the boxes were $16 \times 6 \times 5 \mathrm{~cm}, 12 \times 6$ $\times 5 \mathrm{~cm}$ and $8.6 \times 6 \times 5 \mathrm{~cm}$ for the large, medium and small size respectively. The bottom, left and right sides of each PAO were kept open. The experimental setup of the system is shown in Figure 1.

2.2. Human-Features-Based System Dynamics. According to Figure 2, the targeted dynamics for transferring a PAO horizontally by a subject with the system is described by (1), where $F_{o}=m g$ If we include a hypothesis regarding weight 


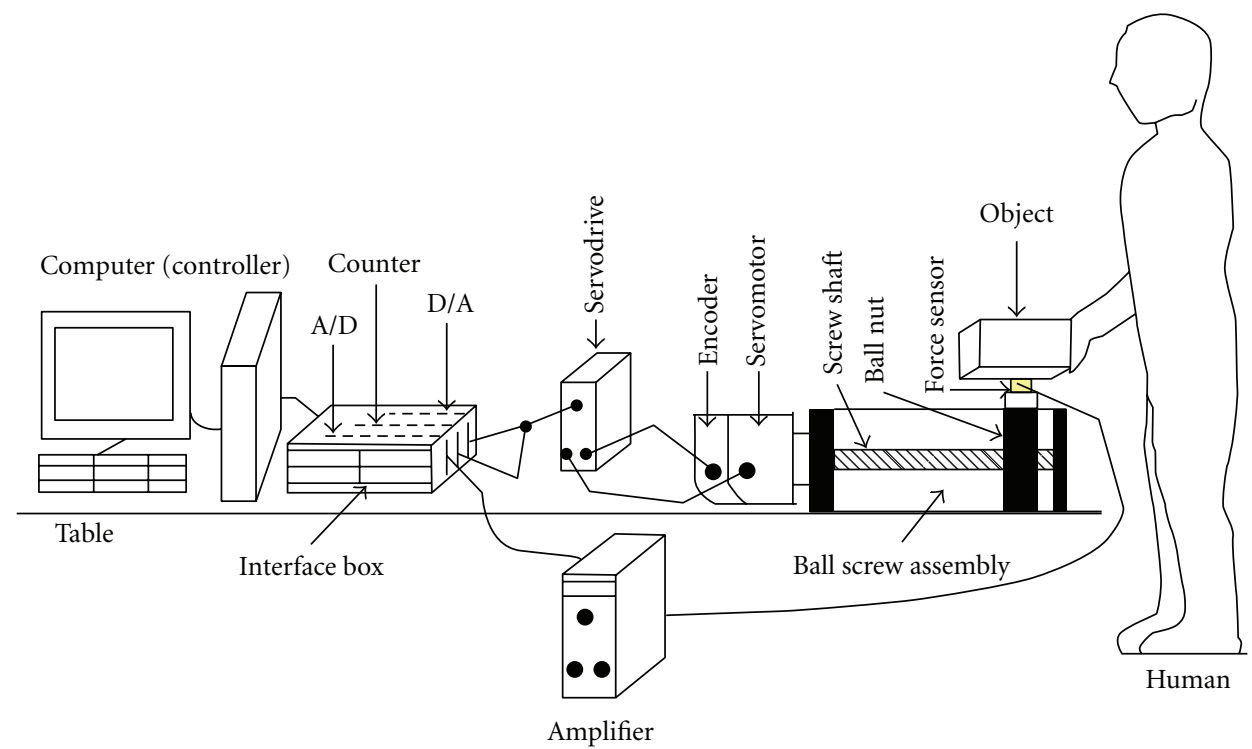

FIGURE 1: Experimental setup of the 1-DOF power assist system for horizontal transfer of objects by the human.

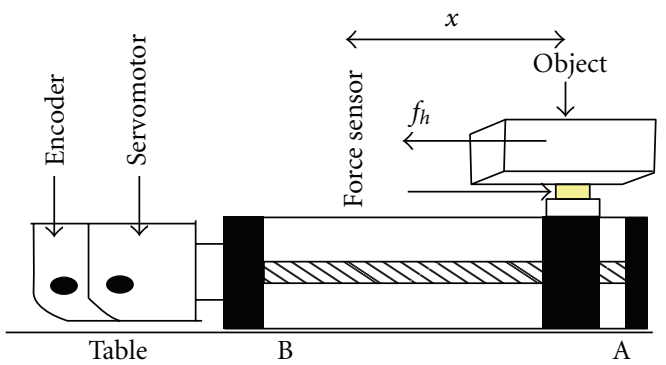

FIGURe 2: Dynamics of the 1-DOF power assist system for horizontal manipulation of objects. The PAO tied to the force sensor is transferred by the subject from position "A" to position "B".

perception in the dynamics, then (1) changes to (2). The hypothesis means that both $m_{1}$ and $m_{2}$ stand for mass, but $m_{1}$ forms inertia force and $m_{2}$ forms gravity force, and $m_{1} \neq m_{2} \neq m, m_{1} \ll m, m_{2} \ll m,\left|m_{1} \ddot{x}_{d}\right| \neq\left|m_{2} g\right|$. A difference between $m_{1}$ and $m_{2}$ is considered due to the difference between human's perception and reality regarding the heaviness of the object transferred with the power assist system [1]. Consider

$$
\begin{aligned}
m \ddot{x_{d}} & =f_{h}+F_{0}, \\
m_{1} \ddot{x_{d}} & =f_{h}+m_{2} g,
\end{aligned}
$$

where, $m=$ actual mass of the PAO, $x_{d}=$ desired displacement of the PAO, $f_{h}=$ load force applied by the subject, $g=$ acceleration of gravity.

2.3. Control System Design. We derived (3) from (2). We then diagrammed the control based on (3), which is shown in Figure 3. If the system is simulated using MATLAB/SIMULINK in velocity control mode of the servomotor, the commanded velocity $\left(\dot{x}_{c}\right)$ to the servomotor is determined by (4), which is fed to the servomotor through a
D/A converter. During simulation, the servodrive determines the error displacement signal by comparing the actual displacement to the desired displacement. Consider

$$
\begin{aligned}
& \ddot{x}_{d}=\frac{1}{m_{1}}\left(f_{h}+m_{2} g\right), \\
& \dot{x}_{c}=\dot{x}_{d}+G\left(x_{d}-x\right) .
\end{aligned}
$$

The control system in Figure 3 is designed for the especially developed 1-DOF power assist system (Figures 1 and 2) for understanding human characteristics and human's interactions with the system. We design the control system shown in Figure 3 following the position control method for controlling the robotic system. The displacement was used to feed-back as it is shown in the figure. Here, the input is the human force $\left(f_{h}\right)$ and the output is the object's displacement. The choice between the position control and the force control is important. We think that the position control is a good choice where the motion path is repeated, wellstructured, well-defined, certain, and the accuracy in the positional movement is desired. On the other hand, the force control may be a good choice where the environment is not well-defined, well-structured, uncertainty is high, motion 


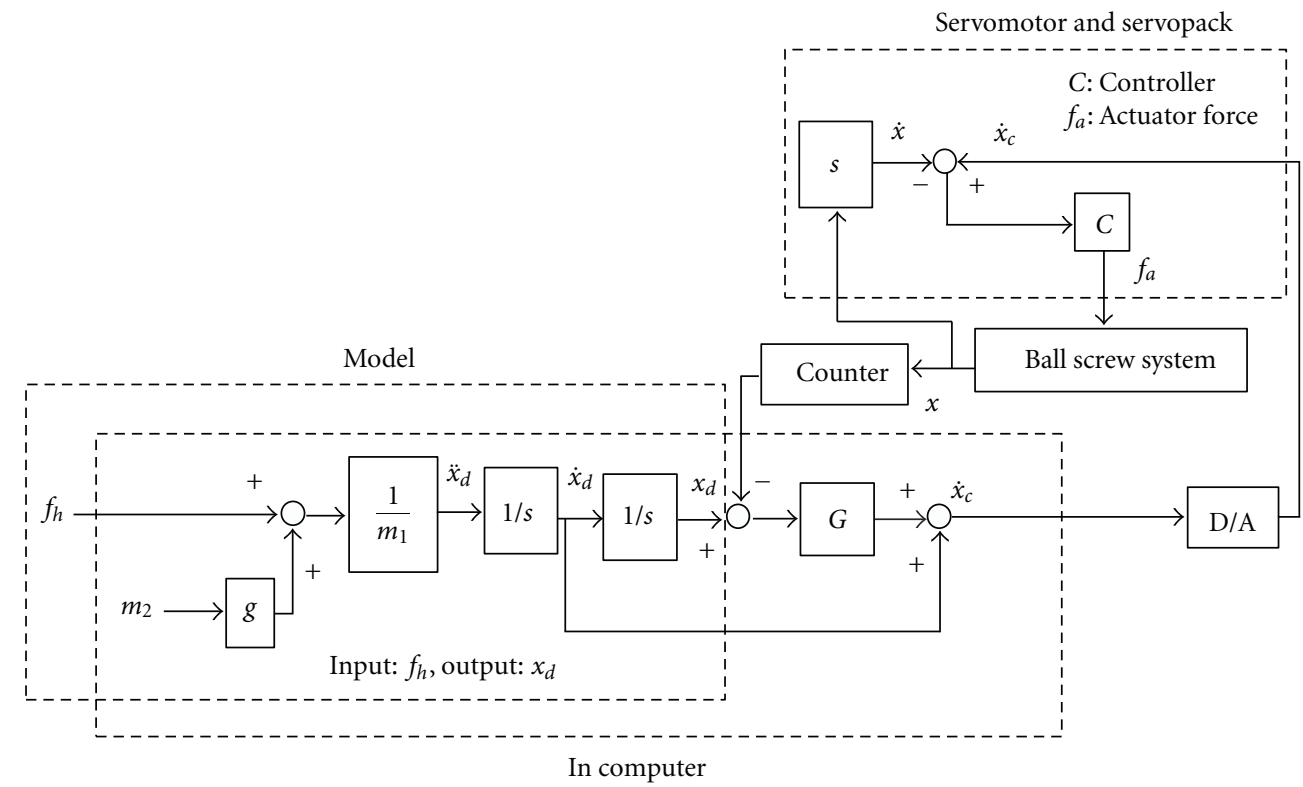

FIGURE 3: Block diagram of the power-assist control. Here, $G$ denotes feedback gain, D/A indicates D/A converter, and $x$ denotes actual displacement. Feedback position control was used with the servomotor in velocity control mode.

path or environment is changeable, and so forth $[10,11,17$, $21,22]$. We used the position control because:

(i) The position control significantly compensates the effects of friction, viscosity, inertia, and so forth. But, these effects need to be considered for the force control though it is very difficult to model and calculate the friction force. Again, the dynamic effects, nonlinear forces, and so forth, affect the system performances for the force control for the multi-degree of freedom systems.

(ii) The actuator force is less and the ball-screw gear ratio is high for the position control. But, the opposite is true for the force control.

(iii) For the position control with high gear ratio, it is easy to realize the real system. But, it is difficult to realize the real system for the force control.

\section{Experiment 1: Analysis of the Human-System Interactions}

We expressed the human's interactions with the system in terms of maneuverability, mobility, naturalness, safety, ease of use, comfort, weight perception, load force, object motion, and so forth, for the objects transferred with the power assist system.

3.1. Experiment Procedures. We nominated ten mechanical engineering male students aged between 23 and 31 years to voluntarily participate in the experiment. We simulated the system shown in Figure 3 using Matlab/Simulink (solver: ode4, Runge-Kutta; type: fixed-step; fundamental sample
TABLE 1: Values of variables for the simulation.

\begin{tabular}{lcccc}
\hline$m_{1}(\mathrm{~kg})$ & 2.0 & 1.5 & 1.0 & 0.5 \\
$m_{2}(\mathrm{~kg})$ & 0.09 & 0.06 & 0.03 & \\
\hline
\end{tabular}

time: $0.001 \mathrm{~s}$ ) for twelve $m_{1}$ and $m_{2}$ sets (Table 1 ) separately. We chose the values of $m_{1}$ and $m_{2}$ based on our experiences.

Each subject transferred each size PAO with the assist system from " $A$ " to "B" as shown in Figure 2 (distance between "A" and "B" was about $0.12 \mathrm{~m}$ ) once for each $m_{1}$ and $m_{2}$ set separately. In each trial, the task required the subject to transfer the object from " $\mathrm{A}$ " to " $\mathrm{B}$ ", maintain the object at " $\mathrm{B}$ " for 1-2 seconds and then release the object. We considered the subject's ease of use and comfort as the evaluation criteria for the maneuverability in transferring objects horizontally with the system [21]. For each trial (for each $m_{1}$ and $m_{2}$ set for each size object), the subject subjectively evaluated (scored) the system for maneuverability from the following alternatives.

(1) Very Easy and Comfortable (score: +2$)$;

(2) Easy and Comfortable (score: +1$)$;

(3) Borderline (score: 0);

(4) Uneasy and Uncomfortable (score: -1$)$;

(5) Very Uneasy and Uncomfortable (score: -2).

All the subjects evaluated the system for maneuverability for the small, medium, and large size objects independently for each $m_{1}$ and $m_{2}$ set. The load force and motion (displacement, velocity, acceleration) data were recorded separately for each trial.

Each subject after each trial also manually transferred a reference-weight object (rectangular box made by bending 
aluminum sheet of thickness $0.5 \mathrm{~mm}$ ) horizontally on a smooth table for about $0.12 \mathrm{~m}$ using the right hand alone for the reference weights. The weight of the reference-weight object could be changed by attaching extra masses inside the box. The subject thus compared the perceived weight of the PAO to that of the reference-weight object and estimated the magnitude of the perceived weight of the PAO following the psychophysical method "constant stimuli". The appearance and size of the PAO were same as that of the reference-weight object for each trial.

\subsection{Experiment Results and Analyses}

3.2.1. Determining Optimum Maneuverability. We determined the mean evaluation scores for the maneuverability for the twelve $m_{1}$ and $m_{2}$ sets for each size object separately. Table 2 shows the mean evaluation scores for the medium size object. We also determined similar scores for the large and small size objects. The results show that the maneuverability is not influenced by the visual object size. The reason may be that the subjects evaluate the maneuverability using their haptic senses where the visual size cues have no or less influence. However, the haptic size cues might influence the maneuverability $[23,24]$.

The results show that ten $m_{1}$ and $m_{2}$ sets got positive scores, but two sets got negative scores. We see that $m_{1}=$ $0.5 \mathrm{~kg}, m_{2}=0.03 \mathrm{~kg}$ and $m_{1}=1 \mathrm{~kg}, m_{2}=0.03 \mathrm{~kg}$ got the highest scores. Hence, the optimum maneuverability is to be achieved at any one of these two conditions. A unique condition for the optimum maneuverability could be determined if we could use more values of $m_{1}$ and $m_{2}$ for the simulation.

The subjects felt very easy and comfortable to manipulate the objects only at $m_{1}=0.5 \mathrm{~kg}, m_{2}=0.03 \mathrm{~kg}$ and $m_{1}=1 \mathrm{~kg}$, $m_{2}=0.03 \mathrm{~kg}$. Hence, we declared these two sets as the optimum conditions for the maneuverability. Here, the optimality/optimization was decided based on the human's feelings following the heuristics. The findings support our hypothesis that we could not identify the positive $m_{1}$ and $m_{2}$ sets (satisfactory maneuverability) from the negative $m_{1}$ and $m_{2}$ sets (unsatisfactory maneuverability) if we did not think $m_{1} \neq m_{2} \neq m, m_{1} \ll m, m_{2} \ll m, m_{1} \ddot{x}_{d} \neq m_{2} g$.

The results show that the optimum/best sets are also the sets of the smallest values of $m_{1}$ and $m_{2}$. Much smaller values of $m_{1}$ and $m_{2}$ may further reduce the perceived heaviness, but it needs to clarify whether or not this is suitable for human psychology. In zero-gravity or weightless condition when $m_{2}=0$, the object is supposed to be too light as it was found in [25] in actual environment and in [26] in virtual environment. But, we previously found that the zerogravity is not feasible because the human loses some haptic information at zero-gravity that reduces human's weight perception ability [23].

3.2.2. Determining Psychophysical Relationships between Actual and Perceived Weights. We determined the mean perceived weight for each size object separately for $m_{1}=0.5 \mathrm{~kg}, m_{2}=$ $0.03 \mathrm{~kg}$ (condition 1) and $m_{1}=1 \mathrm{~kg}, m_{2}=0.03 \mathrm{~kg}$ (condition 2) as presented in Figure 4. We assumed $m_{2}$ as the
TABLE 2: Mean maneuverability scores with standard deviations (in parentheses) for the medium size object.

\begin{tabular}{lcc}
\hline$m_{1}$ & $m_{2}$ & Mean maneuverability score \\
\hline 1 & 0.06 & $+0.83(0.04)$ \\
2 & 0.06 & $+0.33(0.06)$ \\
0.5 & 0.03 & $+2.0(0)$ \\
1 & 0.03 & $+2.0(0)$ \\
1.5 & 0.03 & $+1.5(0.05)$ \\
2 & 0.09 & $-0.17(0.07)$ \\
0.5 & 0.06 & $+1.0(0)$ \\
1.5 & 0.09 & $-0.17(0.08)$ \\
0.5 & 0.09 & $+0.17(0.05)$ \\
1 & 0.09 & $+1.0(0.03)$ \\
1.5 & 0.06 & $+0.67(0.08)$ \\
2 & 0.03 & $+1.17(0.10)$ \\
\hline
\end{tabular}

actual weight of the PAO that is, the actual weight was $0.03 \mathrm{~kg}$ or $0.2943 \mathrm{~N}$ for each size object for two $m_{1}$ and $m_{2}$ sets. We compared the perceived weights of Figure 4 to the actual weight $(0.2943 \mathrm{~N})$ for each size object for $m_{1}=0.5 \mathrm{~kg}$, $m_{2}=0.03 \mathrm{~kg}$ and $m_{1}=1 \mathrm{~kg}, m_{2}=0.03 \mathrm{~kg}$. The figure shows (and we also found in our previous research) that $m_{1}$ does not affect weight perception, but $m_{2}$ does affect [13]. Again, we see that the visual object sizes do not affect weight perception $[13,24]$.

The results for analyses of variances, ANOVAs (visual object size, subject) separately analyzed on the perceived weights for two $m_{1}$ and $m_{2}$ sets showed that the variations due to the object sizes were insignificant $\left(F_{2,18}<1\right.$ for each $m_{1}$ and $m_{2}$ set). The reason may be that the subjects estimated the perceived weights using the haptic cues where the visual cues had no influences [24]. Variations between the subjects were found statistically insignificant $\left(F_{9,18}<1\right.$ for each $m_{1}$ and $m_{2}$ set).

The actual weight of the object was $0.2943 \mathrm{~N}$, but the humans felt about $0.052 \mathrm{~N}$ (Figure 4) when the object was transferred with the system horizontally. Hence, the results show that the perceived weight was about $18 \%$ of the actual weight. Its physical meaning is that the perceived weight of an object transferred with power-assist in the horizontal direction is $18 \%$ of the perceived weight of the same object transferred in the horizontal direction manually. This happens because the power assist system reduces the perceived weight through its assistance to the humans $[1,13]$. It is a known concept that a power assist system reduces the feeling of the weight; however, it was not quantified. This research quantified the weight attenuation for the horizontal transfer of objects with a power assist system. As we compare with our previous research, we find that the perceived weight reduces to $40 \%$ and $20 \%$ of the actual weight if the object is vertically lifted or lowered, respectively $[13,23]$. Weight perception is less for the horizontal manipulation of objects because the gravity force is compensated.

3.2.3. Analysis of Load Force. The time trajectory of the load force for a typical trial is shown in Figure 5. We derived the 


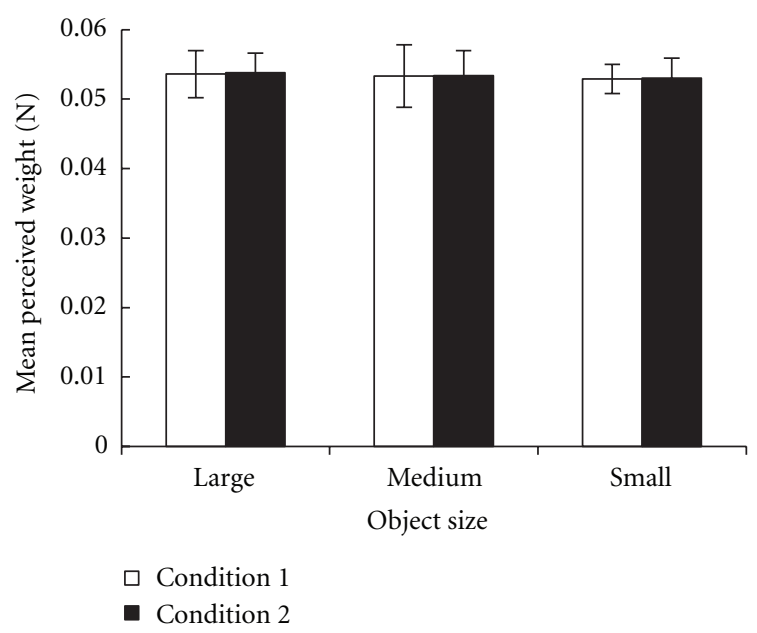

FIGURE 4: Mean $(n=10)$ perceived weights for different object sizes for conditions $1\left(m_{1}=0.5 \mathrm{~kg}, m_{2}=0.03 \mathrm{~kg}\right)$ and $2\left(m_{1}=1 \mathrm{~kg}, m_{2}=\right.$ $0.03 \mathrm{~kg})$.

TABLE 3: Mean peak load forces (PLFs) for different conditions for different object sizes.

\begin{tabular}{lccc}
\hline & \multicolumn{3}{l}{ Mean PLFs $(N)$ with standard deviations (in } \\
$m_{1}, m_{2}$ sets & $\begin{array}{c}\text { parentheses) for different object sizes } \\
\text { Large }\end{array}$ & Medium & Small \\
\hline$m_{1}=0.5 \mathrm{~kg}$, & 2.9131 & 2.6020 & 2.4113 \\
$m_{2}=0.03 \mathrm{~kg}$ & $(0.1307)$ & $(0.1151)$ & $(0.1091)$ \\
\hline$m_{1}=1.0 \mathrm{~kg}$, & 2.9764 & 2.6554 & 2.4602 \\
$m_{2}=0.03 \mathrm{~kg}$ & $(0.1009)$ & $(0.1052)$ & $(0.1067)$ \\
\hline
\end{tabular}

magnitude of the peak load force (PLF) for each object size for conditions $1\left(m_{1}=0.5 \mathrm{~kg}, m_{2}=0.03 \mathrm{~kg}\right)$ and $2\left(m_{1}=\right.$ $1 \mathrm{~kg}, m_{2}=0.03 \mathrm{~kg}$ ) separately and determined the mean PLFs, as shown in Table 3 . We see that the mean PLFs for condition 2 are slightly larger than that for condition 1 . We found previously that $m_{1}$ and $m_{2}$ are linearly proportional to the PLF, and $m_{1}$ affects the load force, but it does not affect the weight perception. On the other hand, $m_{2}$ affects both load force and weight perception [13,23]. We assume that the larger $m_{1}$ in condition 2 has produced the larger PLF.

We have already found in Section 3.2.1 that the subjects feel the best maneuverability at $m_{1}=0.5 \mathrm{~kg}, m_{2}=0.03 \mathrm{~kg}$ and $m_{1}=1 \mathrm{~kg}, m_{2}=0.03 \mathrm{~kg}$. On the other hand, the actually required PLF to transfer the PAO should be slightly larger than the perceived weight [24], which is $0.052 \mathrm{~N}$. We compared the perceived weights from Figure 4 to the PLFs (Table 3) for the large, medium, and small objects and found that the PLFs were very excessive. It means that the subjects apply the load forces that are extremely larger than the actual requirements. We assume that the excessive PLFs cause problems in the human-system interactions in terms of maneuverability, safety, and so forth that we discussed in the introduction. We also see that the magnitudes of the PLFs are proportional to the object sizes $[13,23,24]$.

3.2.4. Analysis of Motions. Figure 5 shows the typical displacement, velocity and acceleration trajectories for a trial.
TABLE 4: Mean peak velocity with standard deviations (in parentheses) for different object sizes for different conditions.

\begin{tabular}{lcc}
\hline \multirow{2}{*}{ Object size } & \multicolumn{2}{c}{ Mean peak velocity $(\mathrm{m} / \mathrm{s})$} \\
& $m_{1}=0.5 \mathrm{~kg}$, & $m_{1}=1.0 \mathrm{~kg}$, \\
$m_{2}=0.03 \mathrm{~kg}$ & $m_{2}=0.03 \mathrm{~kg}$ \\
\hline Large & $0.1497(0.0149)$ & $0.1557(0.0209)$ \\
Medium & $0.1345(0.0157)$ & $0.1399(0.0122)$ \\
Small & $0.1098(0.0121)$ & $0.1176(0.0119)$ \\
\hline
\end{tabular}

We derived the peak velocity and peak acceleration for each trial and determined their means for each object size in each condition separately as given in Tables 4 and 5 respectively. We see in the tables that the velocity and acceleration are large. We assume that the large peak load forces have resulted in the large accelerations that are harmful to the power assist system in terms of maneuverability, safety, and so forth.

\section{Experiment 2: Improving the Human-System Interactions}

Tables 3 and 5 show that the load forces and accelerations are excessive that cause problems in the human-system interactions as we discussed in Section 1. Experiment 2 aimed to design a novel control based on the results of experiment 1 to reduce the excessive load forces and accelerations, and thus to improve the human-system interactions.

4.1. Novel Control Design and Implementation. The novel control was such that the value of $m_{1}$ exponentially declined from a large value to $0.5 \mathrm{~kg}$ when the subject transferred the $\mathrm{PAO}$ with the power assist system and the command velocity of (4) exceeded a threshold. Equations (5) and (6) were used for $m_{1}$ and $m_{2}$, respectively, to augment the effectiveness of the control shown in Figure 3. The novel control is illustrated in Figure 6 as a flowchart. We determined the digit 6 in (5) by trial and error. In fact, the control shown in Figure 3 is 
TABLE 5: Mean peak accelerations with standard deviations (in parentheses) for different object sizes for different conditions.

\begin{tabular}{lcc}
\hline \multirow{2}{*}{ Object size } & \multicolumn{2}{c}{ Mean peak acceleration $\left(\mathrm{m} / \mathrm{s}^{2}\right)$} \\
& $m_{1}=0.5 \mathrm{~kg}$, & $m_{1}=1.0 \mathrm{~kg}$, \\
$m_{2}=0.03 \mathrm{~kg}$ & $m_{2}=0.03 \mathrm{~kg}$ \\
\hline Large & $0.2309(0.0901)$ & $0.2701(0.0498)$ \\
Medium & $0.2282(0.0721)$ & $0.2542(0.0153)$ \\
Small & $0.1887(0.0298)$ & $0.2134(0.0525)$ \\
\hline
\end{tabular}

itself novel as it includes the ideas related to human's weight perception. However, the novelty in the control in Figure 3 is further enhanced as presented in Figure 6 through the exponential reduction of $m_{1}$.

We derived the relationship formula for $m_{1}$ in (5) empirically. The explanation on how to derive the empirical formula is as the following.

(i) Based on the time trajectory of the load force in Figure 5, we derived the magnitude of the peak load force (PLF) for each trial and determined the mean PLFs for each $m_{1}$ and $m_{2}$ set for each object size separately. We then plotted the graph taking the $m_{1}$ values of the twelve $m_{1}$ and $m_{2}$ sets as the abscissa and the mean PLFs for the twelve $m_{1}$ and $m_{2}$ sets for the three objects as the ordinate, and thus determined the relationships between $m_{1}$ and PLFs. The results showed approximately linear relationships between the inertial mass $\left(m_{1}\right)$ and the PLFs.

(ii) We see in Figure 4 (our previously published articles also reported similar results) that humans do not feel the change in $m_{1}$ that is, $m_{1}$ does not affect the haptic perception of weight, but it affects the load force. On the other hand, $m_{2}$ affects both perceived weight and load force $[13,23,27,28]$.

(iii) Based on the information gathered in (i) and (ii), we see that the PLF linearly varies with $m_{1}$. Hence, if we exponentially reduce $m_{1}$ then the PLF will also reduce because it was our goal to reduce the excessive acceleration through the reduction in the excessive PLF. We want to reduce the excessive acceleration because it hampers the human-system interactions. On the other hand, this type of reduction in $m_{1}$ could affect the relationships in (2) that could change in the human's feelings especially the weight perception (in (2), the load force is represented by $f_{h}$ ), but we found empirically in Figure 4 that the reduction (change) in $m_{1}$ does not affect the human's haptic perception of the weight because weight perception is due to $m_{2}$, not due to $m_{1}$. It means that the effect of $m_{1}$ and $m_{2}$ on the human's haptic weight perception is different. Hence, the reduction in $m_{1}$ would also reduce the load force proportionally and the reduction in the load force would not adversely affect the relationships of (2) because the subjects would not feel the change of $m_{1}$. We used this valuable information to model the exponential reduction of $m_{1}$ in (5). However, the magnitudes (digits) used in the formula for $m_{1}$ were determined based on the magnitudes of the PLFs by trial and error. The detailed procedures of deriving the formula are presented in $[13,23,27,28]$ for various conditions. It is also possible to justify the empirical formula using mathematical analysis based on (2).

(iv) The novel control scheme may be considered as an empirically-derived adaptive control method. When the system tends to lose its performances due to the excessive accelerations, the novel control changes itself based on the condition, reduces the accelerations through reducing the load forces, and thus helps the system adapt to the situations [29].

The procedures for experiment 2 were the same as that employed for experiment 1 , but $m_{1}$ and $m_{2}$ were set as $m_{1}=$ $6 * e^{-6 t}+0.5, m_{2}=0.03$ (condition 1.a) and $m_{1}=6 * e^{-6 t}$ $+1.0, m_{2}=0.03$ (condition 2.a) for the simulation. Here, we ignore presenting the simulation details for $m_{1}=6 * e^{-6 t}+$ 1.0, $m_{2}=0.03$ because the concept and procedures for $m_{1}=6$ $* e^{-6 t}+0.5, m_{2}=0.03$ and $m_{1}=6 * e^{-6 t}+1.0, m_{2}=0.03$ are the same:

$$
\begin{gathered}
m_{1}=6 * e^{-6 t}+0.5, \\
m_{2}=0.03 .
\end{gathered}
$$

The performances of the human-robot system were broadly expressed through several criteria such as object motion, object mobility, naturalness, stability, safety, ease of use, and so forth, and in each trial the subject subjectively evaluated (scored) the system using a 7-point bipolar and equal-interval scale as follows.

(1) Best (score: +3$)$;

(2) Better (score: +2$)$;

(3) Good (score: +1$)$;

(4) Alike (score: 0);

(5) Bad (score: -1$)$;

(6) Worse (score: -2$)$;

(7) Worst (score: -3$)$.

\subsection{Evaluation of the Novel Control}

4.2.1. Reduction in PLFs and Peak Accelerations. We compared the mean PLFs for experiment 2 conducted at $m_{1}=$ $6 * e^{-6 t}+0.5, m_{2}=0.03$ and $m_{1}=6 * e^{-6 t}+1.0, m_{2}=0.03$ to that for experiment 1 conducted at $m_{1}=0.5, m_{2}=0.03$ and $m_{1}=1.0, m_{2}=0.03$. The results are shown in Table 6 . The results show that the PLFs reduced significantly due to the novel control.

The mean peak accelerations for different object sizes for experiment 2 are shown in Table 7 . The results show, if we compare these to that of Table 5 , that the peak accelerations reduced due to the application of the novel control. The 

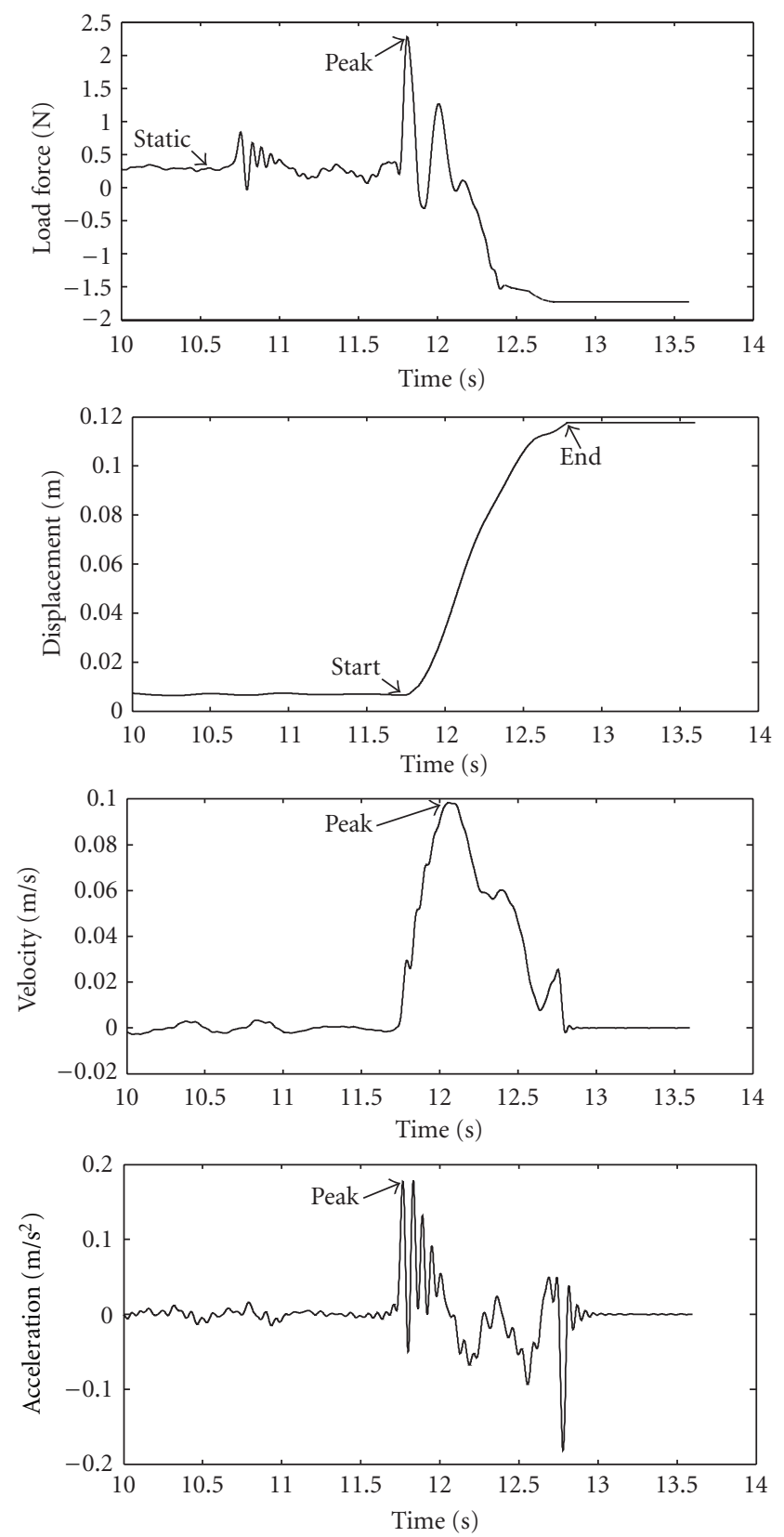

FIGURE 5: Time trajectories of load force, displacement, velocity, and acceleration for a typical trial when a subject transferred the small size $\mathrm{PAO}$ with the system at condition $1\left(m_{1}=0.5 \mathrm{~kg}, m_{2}=0.03 \mathrm{~kg}\right)$.

TABLE 6: Mean peak load forces for different conditions for different object sizes after the application of the novel control.

\begin{tabular}{lccc}
\hline & \multicolumn{3}{c}{ Mean PLFs $(N)$ with standard deviations (in } \\
$m_{1}, m_{2}$ sets & parentheses) for different object sizes \\
& Large & Medium & Small \\
\hline$m_{1}=6 * e^{-6 t}+0.5$, & 1.3569 & 1.1123 & 0.9901 \\
$m_{2}=0.03$ & $(0.0154)$ & $(0.0821)$ & $(0.0910)$ \\
$m_{1}=6 * e^{-6 t}+1.0$, & 1.8646 & 1.5761 & 1.0990 \\
$m_{2}=0.03$ & $(0.0707)$ & $(0.1071)$ & $(0.0885)$ \\
\hline
\end{tabular}

TABle 7: Mean peak accelerations with standard deviations (in parentheses) for different object sizes for different conditions after the application of the novel control.

\begin{tabular}{lcc}
\hline Object size & \multicolumn{2}{c}{ Mean peak acceleration $\left(\mathrm{m} / \mathrm{s}^{2}\right)$ for two $m_{1}, m_{2}$ sets } \\
& $m_{1}=6 * e^{-6 t}+0.5$, & $m_{1}=6 * e^{-6 t}+1.0$, \\
$m_{2}=0.03$ & $m_{2}=0.03$ \\
\hline Large & $0.1234(0.0403)$ & $0.1404(0.0302)$ \\
Medium & $0.1038(0.0233)$ & $0.1220(0.0107)$ \\
Small & $0.0884(0.0111)$ & $0.1008(0.0164)$ \\
\hline
\end{tabular}



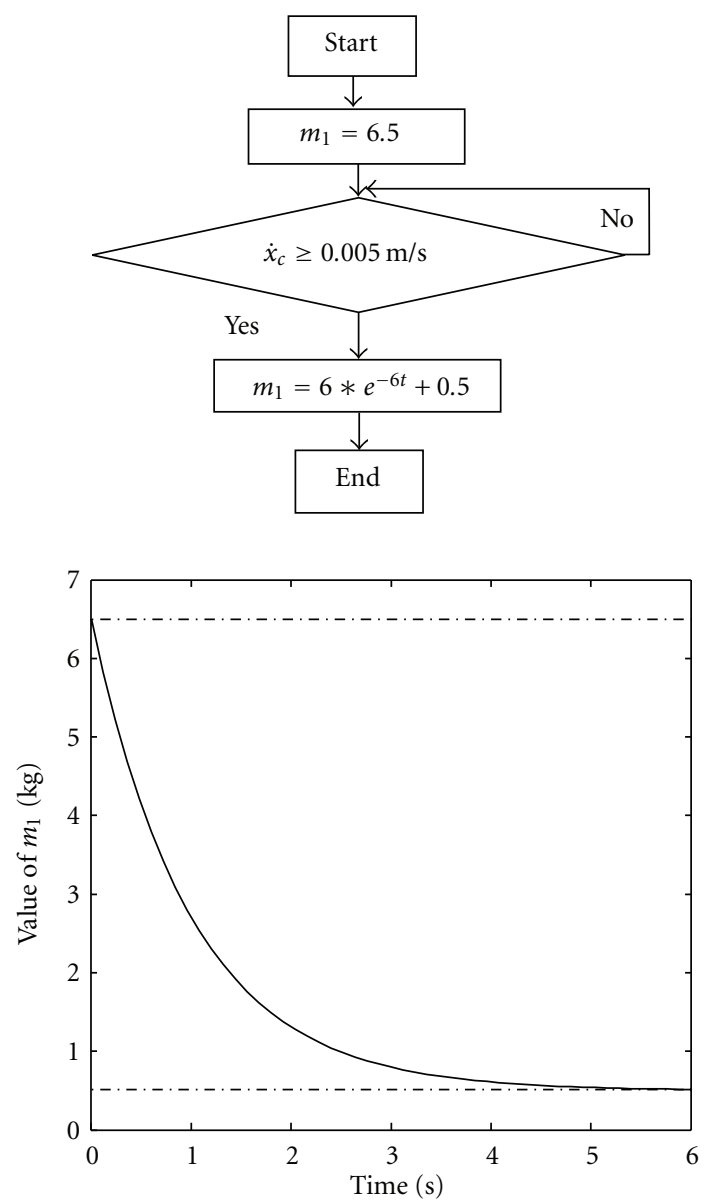

FIGURE 6: Flowchart and hypothetical trajectory of the inertial mass $\left(m_{1}\right)$ for the novel control scheme.

reason may be that the reduced peak load forces due to the novel control reduced the accelerations accordingly. We also found that the velocity reduced slightly due to the novel control.

4.2.2. System Performances Improvement. We determined the mean evaluation scores for the three objects separately. Figure 7 shows the mean evaluation scores for the small size object for two conditions (two $m_{1}, m_{2}$ sets). The scores for the large and medium size objects in each condition were almost the same as that shown in the figure for the small size object. The figure shows that the novel control produced satisfactory system performances.

It is seen in Figure 7 that there is no error bar (individual differences) for the stability and safety, which means that all the subjects evaluated and reported the same score. In fact, the stability and safety were evaluated on whether or not there were any oscillations when transferring the objects with the assist system. No oscillation indicates the stability and the system behaves safe for the user if there is no oscillation. The subjects almost did not report any oscillations during the experiment. This is why all the subjects scored the same value (2.5) for these two criteria. However, there are individual

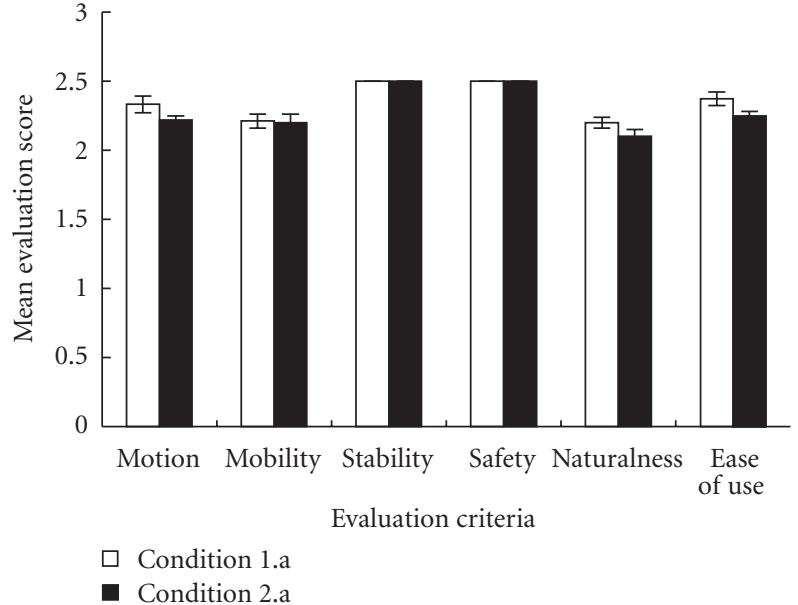

FIGURE 7: Mean performance evaluation scores for the small size object for conditions 1.a $\left(m_{1}=6 * e^{-6 t}+0.5, m_{2}=0.03\right)$ and 2.a $\left(m_{1}=6 * e^{-6 t}+1.0, m_{2}=0.03\right)$ after the application of the novel control.

differences for other criteria. The score 2.5 means that the subject's opinion was between 2 (better) and 3 (best). This special case of evaluation applies to only stability and safety.

We conducted the Analyses of Variances, ANOVAs (object size, subject) on the maneuverability scores, perceived weights, peak load force, peak velocity, peak acceleration, performance evaluation scores, and so forth for experiments 1 and 2 separately. We found that the variations between the object sizes were significant $(P<0.01$ at each case) for the peak load force, peak velocity and peak acceleration. However, the variations between the object sizes were not significant for the maneuverability scores, perceived weights, and performance evaluation scores $(P>0.05$ at each case) [24]. On the other hand, the variations between the subjects were not significant at each case $(P>0.05$ at each case). Hence, the results may be used as the general findings. However, the generality may be increased if we increase the number of trials, object sizes, shapes, subjects, experiment protocols, involvement of the end-users such as the factory people, and so forth.

\section{Discussion}

5.1. Weight Perception in Horizontal Transfer of Objects: A New Initiative. The term weight perception used in this paper combines both the visual (optical) perception and the haptic perception, which involves the tactile perception by the touch through the skin, the proprioceptive perception by the relative position of the grasping parts of the body (fingers), and the kinesthetic perception by the relative movement or motions of the grasping parts of the body (fingers) $[24,30]$. The ideal case or the first type of the weight perception occurs when the human grasps an object and lifts it against the gravity as we studied in [13]. The second type of the weight perception occurs if the human grasps the object and transfers it from one position to another position 
(on a surface), as it is presented in this article $[17,31]$. We considered the second type of the weight perception though this type of weight perception is usually not investigated by other researchers. We, in this paper, investigated the horizontal weight perception because the practical applications of the power assist systems for transferring heavy objects horizontally need to consider this.

5.2. Light-Weight Objects versus Heavy Objects. The optimum/best value of $m_{2}$ (i.e., $m_{2}=0.03 \mathrm{~kg}$ ) derived in this paper does not mean the actual mass of the objects to be transferred in the industrial applications, rather $m_{2}$ means the value that should be put into the control program for getting the optimum maneuverability, safety, stability, and so forth when transferring heavy objects with the power assist systems.

Our ultimate goal is to develop a human-friendly power assist robot system based on the human characteristics for manipulating heavy objects in the industries that would provide satisfactory human-system interactions, as we mentioned in the introduction. However, we could not use a real robotic system, and heavy and large objects. Instead, we used a simulated system, low simulated and actual weights. We think that the following reasons motivated us to use the small and light-weight objects:

(i) we, at this stage, wanted to reduce the costs of developing the real system because a real robotic system convenient for manipulating large and heavy objects is costly;

(ii) we want to compare the findings of this paper to that of other state-of-the-art psychological experiment results, and for this purpose our object sizes and weights need to be small because the psychological tests usually use low weights and small size objects $[24,25,32]$. We think that such comparison with equal basis may produce important information that may help develop the real robotic system in the near future adjusting with the human perceptions such as naturalness, best feelings, and so forth;

(iii) we, in this paper, just wanted to understand human's characteristics and human's interactions with the power assist system in the horizontal object manipulation with the system and then to use the findings (e.g., motivation, problem statement, design ideas, assumptions, hypotheses, dynamic modeling, control programming, novel control strategies, system characteristics reflecting human-system interactions such as relationship between actual and perceived weights, force and motion characteristics, etc.) to develop a real robot capable of manipulating heavy and large objects in the near future. We believe that the findings we have derived will work (but magnitudes may change) for the heavy and large size objects. It may be true that the results are incomplete until we validate those using the heavy and large objects using a real robot. But, it is also true that the results are novel, unique, important, useful and

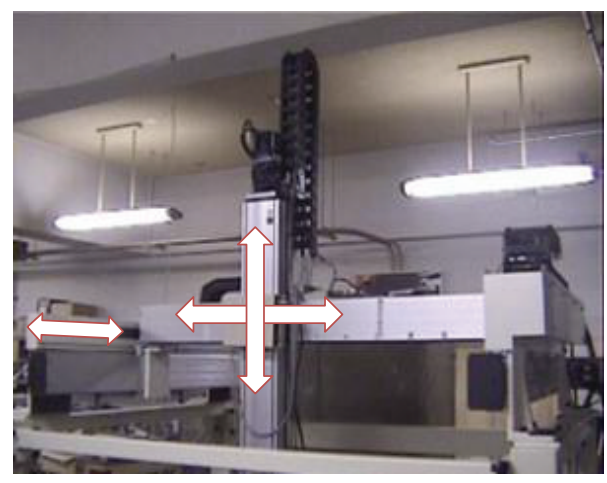

FIGURE 8: The 3-DOF power-assist system for manipulating objects. The arrows show the motion directions.

thus have potential applications for developing real robots for manipulating heavy and large objects in industries. We assume that the physics may behave in the same way when the reinforcement (force support) is a higher magnitude. If not, then the approaches and findings will clearly and definitely guide to develop the real robotic system for manipulating heavy objects. We will report the validation of the results using heavy and large objects and real robots in the forthcoming articles.

5.3. 1-DOF System versus Multi-DOF System. In this paper, we used an especially developed 1-DOF (horizontal motion) power assist system (Figures 1 and 2). The main target of this study was to understand human characteristics and human's interactions with the system for the object manipulation in the horizontal direction. We previously developed similar 1-DOF power assist systems to lift objects in the vertical direction and also conducted numerous studies to understand human characteristics and human's interactions with the system in the vertical lifting of the objects [13, 23, 27, 28]. However, we believe that the findings could be made more practical and accurate if an integrated 2-DOF (or 3-DOF) system consisting of both horizontal and vertical motions could be used for the experiments [31]. Such an integrated 3-DOF system having both horizontal (forward-backward and left-right) and vertical (up-down) motions is shown in Figure 8 . This system may be used to validate the results of this paper. On the other hand, the constraint of the 1-DOF system may affect the subjective results, but the effects are at all not significant as we found in [33].

5.4. Potential of the System to Fulfill the Requirements in Objects Manipulation. The requirements for the successful manipulation of heavy objects with power-assist are as follows: (i) the perceived weight is optimum, (ii) the load force is slightly larger than the perceived weight, (iii) the motions, maneuverability, stability, safety, naturalness, comfort, situational awareness, efficiency, manipulating speed, and so forth, are satisfactory, (iv) the system is enough flexible to adjust with the objects of different sizes, weights, shapes, and so forth, (v) the objects can be manipulated in various 
degrees of freedoms for example, vertical, horizontal, and so forth, (vi) the system produces satisfactory performances even in the worst-cases, uncertain, rapid changing situations, disturbances, and so forth. The proposed system along with its previous works and future extensions may satisfy these requirements $[12,13,23]$.

5.5. Effectiveness and Accuracy of the Results. The servomotor was kept in the velocity control mode. Another mode, the torque control mode, may be tested to verify the results. The results do not violate the size-weight illusion because the objects of different sizes were handled independently [32]. The evaluation methodologies of the human factors (e.g., weight perception) are subjective instead of objective. Nevertheless, the subjective evaluation is to be reliable because the subjective evaluations in the technical domains have already been proven effective and reliable in many cases [34]. However, accuracy of the findings may be enhanced by transforming the maneuverability evaluation scale from 5-point to 7-point and by improving the quality of the evaluation alternatives and evaluation criteria and by increasing the number of the subjects and the trials.

\section{Conclusions and Future Works}

In this paper, we successfully presented a 1-DOF power assist robot system for transferring objects by the human subjects in the horizontal direction. We included human features (e.g., weight perception) in the robot dynamics and control. We simulated the system and analysed the human-system interactions such as we determined the optimum manoeuvrability conditions for transferring objects with it. We also determined the psychophysical relationships between the actual and the perceived weights for the objects transferred with the system. We analyzed weight perception, load forces and motion characteristics, and so forth. We then used the findings to develop a novel biomimetic control method for the robot. The novel control was implemented and it was found improving the human-system interactions in terms of object mobility, safety, naturalness, maneuverability, and so forth. The novel control was designed following the biomimetic or the human-interactive approaches, and psychophysics was used that determined the relationships between the physical stimuli and the sensory responses [24, $35,36]$.

The findings may help develop human-friendly power assist devices for handling heavy objects in industries such as manufacturing and assembly, mining, logistics and transport, construction, agriculture, and so forth. The findings are novel in the sense that the human cues are included in the robot dynamics and control, and a weight-perceptionbased model of the horizontal transfer of objects with powerassist is presented that was neither previously addressed by other researchers nor considered in our previous works. The findings may enhance the state-of-the-art knowledge and applications of psychology, robotics, biomimetics, control system, automation, human factors, HCI, HRI, interface design and evaluation, interactive system design, and so forth.

We will verify the results using heavy objects and real robotic systems in the near future. The system will be upgraded to a real multi-degree of freedom system and it will be evaluated properly for heavy objects. We will enhance the compliance of the actuation [37]. More human features will be investigated and be used to design the control to further improve the human-system interactions. The biomimetic and the psychophysical approaches to the control design will be considered for other assistive and interactive applications such as rehabilitation, healthcare, and so forth $[22,35,37-$ 39].

\section{Acknowledgment}

The authors express their thanks and gratitude to the Japanese Ministry of Education, Culture, Sports, Science, and Technology.

\section{References}

[1] H. Kazerooni, "Extender: a case study for human-robot interaction via transfer of power and information signals," in Proceedings of the 2nd IEEE International Workshop on Robot and Human Communication, pp. 10-20, November 1993.

[2] K. Kong, H. Moon, B. Hwang, D. Jeon, and M. Tomizuka, "Impedance compensation of SUBAR for back-drivable forcemode actuation," IEEE Transactions on Robotics, vol. 25, no. 3 , pp. 512-521, 2009.

[3] H. Seki, K. Ishihara, and S. Tadakuma, "Novel regenerative braking control of electric power-assisted wheelchair for safety downhill road driving," IEEE Transactions on Industrial Electronics, vol. 56, no. 5, pp. 1393-1400, 2009.

[4] T. Kawashima, "Study on intelligent baby carriage with power assist system and comfortable basket," Journal of Mechanical Science and Technology, vol. 23, no. 4, pp. 974-979, 2009.

[5] T. Tanaka, Y. Satoli, S. Kaneko, Y. Suzuki, N. Sakamoto, and S. Seki, "Smart suit: Soft power suit with semi-active assist mechanism - Prototype for supporting waist and knee joint," in Proceedings of the International Conference on Control, Automation and Systems (ICCAS '08), pp. 2002-2005, October 2008.

[6] G. Q. Liu, Y. C. Yan, J. Chen, and T. M. Na, "Simulation and experimental validation study on the drive performance of a new hydraulic power assist system," in Proceedings of the IEEE Intelligent Vehicles Symposium, pp. 966-970, June 2009.

[7] S. Lee, S. Hara, and Y. Yamada, "Safety-preservation oriented reaching monitoring for smooth control mode switching of skill-assist," in Proceedings of the IEEE International Conference on Systems, Man and Cybernetics (SMC '08), pp. 780-785, October 2008

[8] K. Osamura, S. Kobayashi, M. Hirata, and H. Okamoto, "Power assist control for slide doors using an ideal door model," in Proceedings of the IEEE International Symposium on Industrial Electronics (ISIE '08), pp. 1293-1299, July 2008.

[9] K. Kosuge, H. Yabushita, and Y. Hirata, "Load-free control of power-assisted cycle," in Proceedings of the 1st IEEE Technical Exhibition Based Conference on Robotics and Automation (TExCRA '04), pp. 111-112, November 2004.

[10] M. Ding, J. Ueda, and T. Ogasawara, "Pinpointed muscle force control using a power-assisting device: system configuration 
and experiment," in Proceedings of the 2nd Biennial IEEE/RASEMBS International Conference on Biomedical Robotics and Biomechatronics (BioRob '08), pp. 181-186, October 2008.

[11] T. Kusaka, T. Tanaka, S. Kaneko, Y. Suzuki, M. Saito, and H. Kajiwara, "Assist force control of smart suit for horse trainers considering motion synchronization," International Journal of Automation Technology, vol. 3, no. 6, pp. 723-730, 2009.

[12] S. M. M. Rahman, R. Ikeura, M. Nobe, and H. Sawai, "Design of a power assist system for lifting objects based on human's weight perception and changes in system's time constant," in Proceedings of the 2nd Conference on Human System Interactions, (HSI '09), pp. 664-671, Catania, Italy, May 2009.

[13] S. M. M. Rahman, R. Ikeura, M. Nobe, and H. Sawai, "Design and control of a 1DOF power assist robot for lifting objects based on human operator's unimanual and bimanual weight discrimination," in Proceedings of the IEEE International Conference on Mechatronics and Automation (ICMA '09), pp. 3637-3644, August 2009.

[14] M. M. Ayoub, "Problems and solutions in manual materials handling: the state of the art," Ergonomics, vol. 35, no. 7-8, pp. 713-728, 1992.

[15] A. M. Okamura, N. Smaby, and M. R. Cutkosky, "Overview of dexterous manipulation," in Proceedings of the IEEE International Conference on Robotics and Automation (ICRA '00), pp. 255-262, April 2000.

[16] T. Doi, H. Yamada, T. Ikemoto, and H. Naratani, "Simulation of a pneumatic hand crane power-assist system," Journal of Robotics and Mechatronics, vol. 20, no. 6, pp. 896-902, 2008.

[17] S. Hara, "A smooth switching from power-assist control to automatic transfer control and its application to a transfer machine," IEEE Transactions on Industrial Electronics, vol. 54, no. 1, pp. 638-650, 2007.

[18] T. Takubo, H. Arai, K. Tanie, and T. Arai, "Human-robot cooperative handling using variable virtual nonholonomic constraint," International Journal of Automation Technology, vol. 3, no. 6, pp. 653-662, 2009.

[19] A. Niinuma, T. Miyoshi, K. Terashima, and Y. Miyashita, "Evaluation of effectiveness of a power-assisted wire suspension system compared to conventional machine," in Proceedings of the IEEE International Conference on Mechatronics and Automation (ICMA '09), pp. 369-374, August 2009.

[20] T. Miyoshi and K. Terashima, "Study on horizontal power assisted system for overhead crane," International Journal of Applied Electromagnetics and Mechanics, vol. 24, no. 3-4, pp. 297-309, 2006.

[21] H. Seki, M. Iso, and Y. Hori, "How to design force sensorless power assist robot considering environmental characteristics-position control based or force control based," in Proceedings of the 28th Annual Conference of the IEEE Industrial Electronics Society, pp. 2255-2260, November 2002.

[22] L. Vanacken, R. Pinho, J. Sijbers, and K. Coninx, "Force feedback to assist active contour modelling for tracheal stenosis segmentation," Advances in Human-Computer Interaction, vol. 2012, Article ID 632498, 9 pages, 2012.

[23] S. M. M. Rahman, R. Ikeura, M. Nobe, and H. Sawai, "Controlling a power assist robot for lifting objects considering human's unimanual, bimanual and cooperative weight perception," in Proceedings of the IEEE International Conference on Robotics and Automation (ICRA '10), pp. 2356-2362, May 2010.

[24] A. M. Gordon, H. Forssberg, R. S. Johansson, and G. Westling, "Visual size cues in the programming of manipulative forces during precision grip," Experimental Brain Research, vol. 83, no. 3, pp. 477-482, 1991.
[25] M. O. Ernst and M. S. Banks, "Humans integrate visual and haptic information in a statistically optimal fashion," Nature, vol. 415, no. 6870, pp. 429-433, 2002.

[26] L. Dominjon, A. Lécuyer, J. M. Burkhardt, P. Richard, and S. Richir, "Influence of control/display ratio on the perception of mass of manipulated objects in virtual environments," in Proceedings of the IEEE Virtual Reality (VR '05), pp. 19-25, March 2005.

[27] S. M. M. Rahman, R. Ikeura, M. Nobe, and H. Sawai, "Control of a power assist robot for lifting objects based on human operator's perception of object weight," in Proceedings of the 18th IEEE International Symposium on Robot and Human Interactive Communication (RO-MAN '09), pp. 84-90, Toyama, Japan, October 2009.

[28] S. M. M. Rahman, R. Ikeura, S. Hayakawa, and H. Sawai, “A critical look at human's bimanual lifting of objects with a power assist robot and its applications to improve the powerassist control," in Proceedings of the IEEE International Conference on Robotics and Biomimetics (ROBIO '10), pp. 732-738, Tianjin, China, December 2010.

[29] K. Astrom, Adaptive Control, Dover, New York, NY, USA, 2008.

[30] D. Hecht and M. Reiner, "Stroop interference and facilitation effects in kinesthetic and haptic tasks," Advances in HumanComputer Interaction, vol. 2010, Article ID 852420, 2010.

[31] S. M. M. Rahman, R. Ikeura, M. Nobe, and H. Sawai, "Study on optimum maneuverability in horizontal manipulation of objects with power-assist based on weight perception," in Mechatronics and Information Technology (ICMIT '09), vol. 7500 of Proceedings of SPIE, December 2009.

[32] J. R. Flanagan and M. A. Beltzner, "Independence of perceptual and sensorimotor predictions in the size-weight illusion," Nature Neuroscience, vol. 3, no. 7, pp. 737-741, 2000.

[33] S. M. Mizanoor Rahman, R. Ikeura, I. Shinsuke, S. Hayakawa, and H. Sawai, "Psychophysical relationships between actual and perceived weights for lifting objects with power-assist: consideration of constrained and unconstrained lifting," in Proceedings of the 3rd International Symposium on System Integration (SII '10), pp. 152-157, Tohoku University, Sendai, Japan, December 2010.

[34] H. Kobayashi, R. Ikeura, and H. Inooka, "Evaluating the maneuverability of a control stick using electromyography," Biological Cybernetics, vol. 75, no. 1, pp. 11-18, 1996.

[35] H. L. Xie, Z. Z. Liang, F. Li, and L. X. Guo, "The knee joint design and control of above-knee intelligent bionic leg based on magneto-rheological damper," International Journal of Automation and Computing, vol. 7, no. 3, pp. 277-282, 2010.

[36] T. J. Li, G. Q. Chen, and G. F. Shao, "Action control of soccer robots based on simulated human intelligence," International Journal of Automation and Computing, vol. 7, no. 1, pp. 55-63, 2010.

[37] N. Costa, M. Bezdicek, M. Brown, J. Gray, and D. Caldwell, "Joint motion control of a powered lower limb orthosis for rehabilitation," International Journal of Automation and Computing, vol. 3, no. 3, pp. 271-281, 2006.

[38] J. Boeck, L. Vanacken, S. Notelaers, and K. Coninx, "Improved haptic linear lines for better movement accuracy in upper limb rehabilitation," Advances in Human-Computer Interaction, vol. 2012, Article ID 162868, 7 pages, 2012.

[39] H. Mi, A. Krzywinski, T. Fujita, and M. Sugimoto, "Robotable: an infrastructure for intuitive interaction with mobile robots in a mixed-reality environment," Advances in HumanComputer Interaction, vol. 2012, Article ID 301608, 10 pages, 2012. 

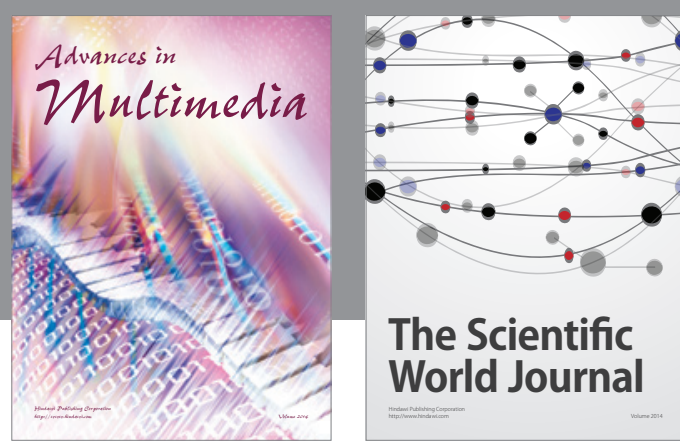

The Scientific World Journal
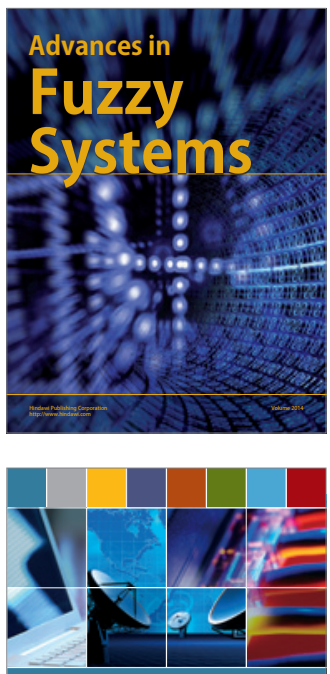

Computer Networks and Communications
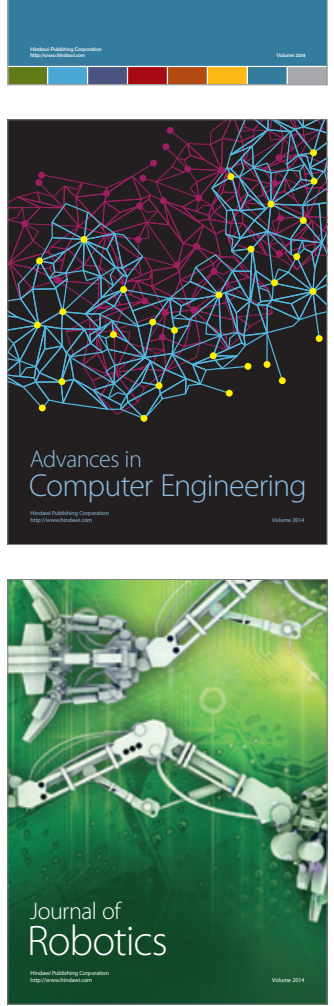
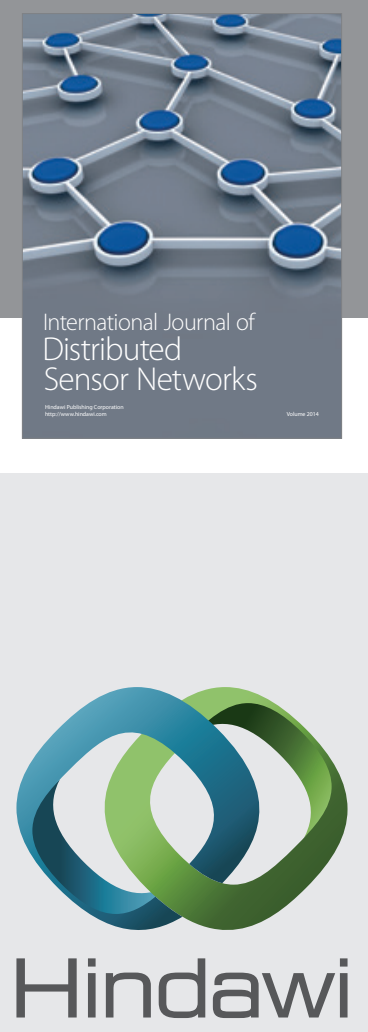

Submit your manuscripts at

http://www.hindawi.com
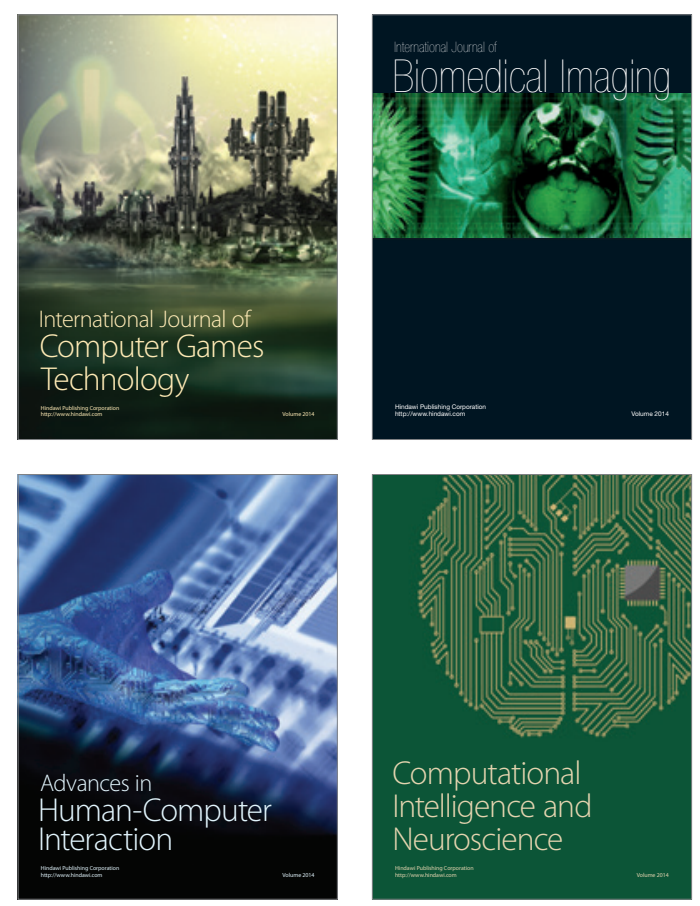
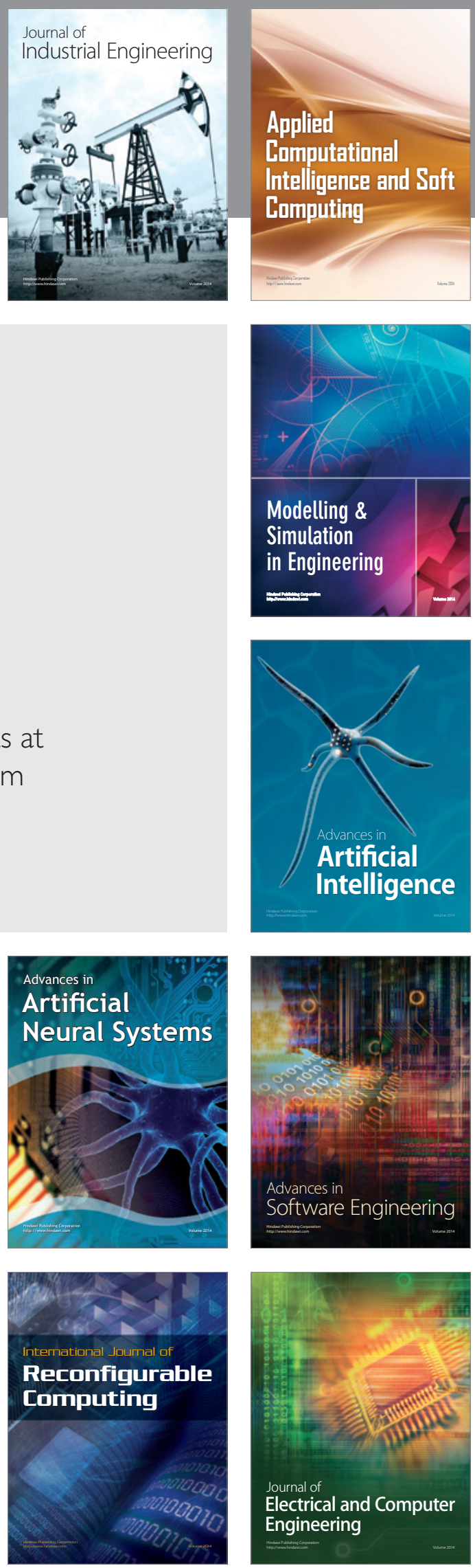